\title{
Differential expressions of integrin-linked kinase, $\beta$-parvin and cofilin 1 in high-fat diet induced prostate cancer progression in a transgenic mouse model
}

\author{
MENG-BO HU ${ }^{1 *}$, JI-MENG HU $^{1 *}$, LI-REN JIANG ${ }^{2}$, TIAN YANG ${ }^{1}$, WEN-HUI ZHU ${ }^{1}$, \\ YUN HU ${ }^{1}$, XIAO-BO WU ${ }^{1}$, HAO-WEN JIANG ${ }^{1}$ and QIANG DING ${ }^{1}$ \\ ${ }^{1}$ Department of Urology, Huashan Hospital, Fudan University, Shanghai 200040; ${ }^{2}$ Department of Pathology, \\ Shanghai General Hospital, Shanghai Jiaotong University, School of Medicine, Shanghai 201620, P.R. China
}

Received January 19, 2018; Accepted August 1, 2018

DOI: $10.3892 / \mathrm{ol} .2018 .9276$

\begin{abstract}
High-fat diet induced obesity was associated with more aggressive prostate cancer. Recent research has demonstrated that integrin-linked kinase (ILK), $\beta$-parvin and downstream cofilin 1 jointly affected cancer progression. Meanwhile, these proteins were also involved in energy metabolism. Therefore, the present study was conducted to investigate the potential function of ILK, $\beta$-parvin and cofilin 1 in the high-fat diet-induced progression of prostate cancer. Transgenic mice with prostate cancer were employed, fed with different diets and sacrificed at 20 and 28 weeks. Tumor differentiation, extracapsular extension and metastasis were compared between the groups. Expression levels of ILK, $\beta$-parvin and cofilin 1 in prostate were evaluated by immunohistochemical analysis and determined by an immunoreactivity score. Public databases were applied for analysis and validation. It was detected that high-fat diet feeding promoted cancer progression in transgenic mice with prostate cancer, with increased expressions of $\beta$-parvin $(\mathrm{P}=0.038)$ and cofilin $1(\mathrm{P}=0.018)$. Higher expressions of ILK, $\beta$-parvin and cofilin 1 were also associated with poorer cancer differentiation. Additionally, higher mRNA levels of CFL1 were correlated with a worse disease-free survival in patients of certain subgroups from The Cancer Genome Atlas database. Further studies were warranted in discussing the potential roles of ILK, $\beta$-parvin and cofilin 1 in high-fat diet feeding induced progression of prostate cancer.
\end{abstract}

Correspondence to: Dr Hao-Wen Jiang, Department of Urology, Huashan Hospital, Fudan University, 12 Middle Wulumuqi Road, Shanghai 200040, P.R. China

E-mail: oncouro_jhw@126.com

${ }^{*}$ Contributed equally

Key words: $\beta$-parvin, cofilin 1, high-fat diet, integrin-linked kinase, prostate carcinoma

\section{Introduction}

Prostate cancer $(\mathrm{PCa})$ recently ranked as the second most diagnosed malignancy and the fifth leading cause of cancer death in men globally (1). Its development and progression were closely associated with another global epidemic, obesity, as extensive evidence showed up demonstrating various epidemiological and biological associations (2). Obesity was proved to be associated with more aggressive PCa, e.g., higher pathological grade (3), higher recurrence rate after definitive therapy (4), and higher cancer-specific mortality (5). However, the exact molecular mechanisms contributing to obesity induced PCa progression remained largely unclear.

The progression and metastasis of cancer required cell mobilization and epithelial mesenchymal transition (EMT), involving the filopodium-like protrusions (FLPs) of cancer cells that interacted productively with surrounding microenvironment (6). The researchers identified that the activation of integrin-linked kinase (ILK), $\beta$-parvin, cofilin pathway could promote caner progression, via enhancing the formation of FLPs and maintaining its existence, which raised a brand new perspective in cancer researches (6). Meanwhile, the signaling of ILK, $\beta$-parvin or cofilin were also involved in obesity and energy metabolism as recently proposed (7-9). Liu et al (7) discovered that oleic acid, with high levels in sera of obese patients, would activate ILK signaling pathway and therefore promote proliferation of renal cell carcinoma. It was also demonstrated that ILK might promote diet-induced insulin resistance in obese mice, by impairing insulin signaling and insulin perfusion through capillaries via ILK-PINCH-parvin complex (IPP) (8). Besides, Cofilin 1 (CFL1) gene expression was proved to be markedly elevated in patients with metabolic syndrome in Turkish population (9). Despite the complicated crosstalk and molecular network, these findings guided us to explore whether ILK/ $\beta$-parvin/cofilin pathway played a role in obesity induced cancer progression.

To better elucidate the effect of obesity on development and progression of $\mathrm{PCa}$, we applied high-fat diet (HFD) to induce obesity in transgenic adenocarcinoma of mouse prostate (TRAMP) animal model, which was considered the best model to resemble the natural process of PCa progression in obese subjects (10). We aimed to verify the hypothesis that 
ILK/ $\beta$-parvin/cofilin pathway would affect the cancer progression in obese patients. With public database, we endeavored to further validate the differential expressions of ILK, $\beta$-parvin and cofilin in $\mathrm{PCa}$, investigate their roles in cancer survival, and explore the possible molecular network.

\section{Materials and methods}

Animals and diets. The present study was carried out in strict accordance with the National Institutes of Health guide for the care and use of Laboratory animals (NIH Publications no. 8023, revised 1978). The protocol was approved by the Institutional Animal Care and Use Committee from Department of Laboratory Animal Science, Fudan University (20160816A197). The TRAMP mice were obtained from Jackson Laboratory (Bar Harbor, Maine, USA), bred and maintained under specific pathogen free conditions (SPF, Grade III) at Department of Laboratory Animal Science, Fudan University (Shanghai, China, certificate no. SCXK-HU-2014-0004). Each mouse was kept separately in a cage, bedding of cork dust, with a 12-hour light-dark cycle. All male TRAMP mice were selected by genotyping, and randomly admitted to two groups where the mice were fed with micronutrients-matched control diet (CD) or HFD ad libitum at 5 weeks of age as previously described (11). CD (16\% calories from fat) and HFD (40\% calories from fat) was supplied by Puluteng Bio-technology, Shanghai, China. TRAMP mice were sacrificed at 20 or 28 weeks of the age, resulting in a total of four groups in the present study (CD-fed 20-week TRAMP, n=12; HFD-fed 20-week TRAMP, $n=12$; CD-fed 28-week TRAMP, n=12; HFD-fed 28-week TRAMP, $\mathrm{n}=12$ ). The maximum diameter of tumor allowed was $1.5 \mathrm{~cm}$, and none of tumors in the present study reached this diameter.

Systemic evaluation and tissue preparation. All TRAMP mice received body weight and blood glucose examinations before sacrifice. Then, the mouse underwent general anesthesia with intraperitoneal injection of pentobarbital $(50 \mathrm{mg} / \mathrm{kg})$, scanned by GE eXplore Locus micro-CT scanner (GE Healthcare Biosciences, Chicago, IL, USA) for systemic evaluation, and euthanized by asphyxiation of $\mathrm{CO}_{2}$ (flow rate at $1.5 \mathrm{l} / \mathrm{min}$ ). The prostate tumor, genitourinary tract, epididymal fat, enlarged lymph nodes, liver and lung were removed from the mouse, weighed, and fixed for further analysis.

Pathological and immunohistochemical analysis. Prostate and other prepared tissues were fixed in $10 \%$ buffered formalin, processed in an alcohol-xylene series, and embedded in paraffin. A series of sections were prepared with hematoxylin and eosin staining, for evaluation of tumor differentiation, extracapsular extension and confirmation of distant metastasis. The IHC was performed in mouse prostate. All prostate sections were dewaxed, rehydrated and incubated in $3 \%$ hydrogen peroxide for $10 \mathrm{~min}$ at room temperature to block the activity of endogenous peroxidase. These sections were then incubated overnight at $4^{\circ} \mathrm{C}$ with rabbit monoclonal Anti-ILK, Anti-B-Parvin and Anti-Cofilin antibody, respectively (dilution, 1:50; Abcam, Cambridge, MA, USA). Staining was detected using Envision detection kit (Dako, Hamburg, Germany) and diaminobenzidine (DAB) as the chromogen, according to the manufacturer's instructions. In each mouse, three sections of the prostate were included for further evaluation and analysis.

Evaluation of sections were accomplished by two pathologists at our institute. Immunoreactivity score (IRS) category, determined by the multiplication of score of staining intensity and score of percentage of positive cells, was applied to assess the immunohistochemistry (IHC) sections as previously described (12). In specific, intensity of staining was classified as 0 (negative), 1 (weak), 2 (moderate), and 3 (strong), and percentage of positive cells was classified as $0(<1 \%), 1(1-25 \%)$, $2(26-50 \%), 3(51-75 \%)$, and $4(76-100 \%)$. IRS category was thus determined as score 0 (negative, multiplication values 0 ), score 1 (weakly positive, multiplication values 1,2 ), score 2 (moderately positive, multiplication values $3,4,6$ ) and score 3 (strongly positive, multiplication values $8,9,12$ ).

Public database analysis and validation. The molecular and clinical data of prostate adenocarcinoma patients (499 samples) were obtained from The Cancer Genome Atlas (TCGA) database that generated by TCGA Research Network (http://cancergenome.nih.gov/). Using cBioPortal for Cancer Genomics (http://www.cbioportal.org) $(13,14)$, these data were further analyzed in Oncoprint, disease-free survival (DFS), and biological neighborhood networks, to validate and explore the expressions of ILK, $\beta$-parvin and cofilin in PCa patients.

Statistical analysis. The results were presented as mean \pm standard error. TRAMP mice sample size was determined by previous experiments on the characterization of the mouse model, and all mice were randomized to different groups in a blinded manner. Unpaired two-tailed Student's t-test, analysis of variance, Fisher exact test, Pearson's correlation test or Kaplan-Meier curves with log-rank test were conducted, as appropriate, using SPSS Statistics version 24 (IBM, Armonk, NY, USA) and Prism version 6.0c (GraphPad Software, La Jolla, CA, USA). A two-tailed $\mathrm{P}<0.05$ was considered to indicate a statistically significant difference in all analyses.

Data availability. The datasets generated and analyzed during the current study are available from the corresponding author on reasonable request.

\section{Results}

$H F D$ feeding stimulated fat accumulation and promote $P C a$ progression in TRAMP mice. HFD feeding stimulated adipose tissue accumulation and increased body weight in TRAMP mice, as presented in Table I. In 28-week group, the body weight of mice fed with HFD was higher than that fed with CD (34.2 g vs. 28.2 g, P<0.001). Meanwhile, the genitourinary weight was also higher in HFD-fed mice in 28-week group (1.54 g vs. $1.21 \mathrm{~g}, \mathrm{P}<0.05)$. The epididymal fat weight was higher in HFD-fed mice in both 20 -week $(1.05 \mathrm{~g}$ vs. $0.53 \mathrm{~g}$, $\mathrm{P}<0.01)$ and 28 -week group (1.32 g vs. $0.58 \mathrm{~g}, \mathrm{P}<0.01)$.

We further determined the development and progression of PCa, by evaluating the tumor differentiation, extracapsular extension and metastasis (Table I). PCa formation was detected from all TRAMP mice in the present study. Compared with 
Table I. Comparisons of systemic characteristics and tumor progression between control diet-fed and high-fat diet-fed TRAMP mice.

\begin{tabular}{|c|c|c|c|c|}
\hline \multirow[b]{2}{*}{ Variables } & \multicolumn{2}{|c|}{ 20-week } & \multicolumn{2}{|c|}{ 28-week } \\
\hline & $\begin{array}{l}\text { Control diet } \\
\qquad(\mathrm{N}=12)\end{array}$ & $\begin{array}{l}\text { High-fat diet } \\
\qquad(\mathrm{N}=12)\end{array}$ & $\begin{array}{l}\text { Control diet } \\
\qquad(\mathrm{N}=12)\end{array}$ & $\begin{array}{l}\text { High-fat diet } \\
\qquad(\mathrm{N}=12)\end{array}$ \\
\hline Body weight $^{\text {a }}(\mathrm{g})$ & $20.8 \pm 0.52$ & $22.3 \pm 1.1$ & $28.2 \pm 0.61$ & $34.2 \pm 1.44^{\mathrm{f}}$ \\
\hline Blood glucose $^{\mathrm{a}}(\mathrm{mmol} / \mathrm{l})$ & $15.1 \pm 0.95$ & $15.2 \pm 1.41$ & $14.8 \pm 1.07$ & $18.8 \pm 1.85$ \\
\hline Genitourinary weight ${ }^{\mathrm{a}}$ (g) & $0.86 \pm 0.06$ & $1.02 \pm 0.08$ & $1.21 \pm 0.07$ & $1.54 \pm 0.13^{\mathrm{d}}$ \\
\hline Epididymal fat weight ${ }^{\mathrm{a}}(\mathrm{g})$ & $0.53 \pm 0.09$ & $1.05 \pm 0.14^{\mathrm{e}}$ & $0.58 \pm 0.1$ & $1.32 \pm 0.22^{\mathrm{e}}$ \\
\hline Tumor diameter ${ }^{\mathrm{a}}(\mathrm{mm})$ & $6.17 \pm 0.19$ & $6.08 \pm 0.17$ & $7.67 \pm 0.16$ & $8.17 \pm 0.25$ \\
\hline \multicolumn{5}{|l|}{ Tumor differentiation $(\%)$} \\
\hline Well & 50.0 & 41.7 & 8.3 & 8.3 \\
\hline Moderate & 25.0 & 33.3 & 33.3 & 16.7 \\
\hline Poor & 25.0 & 25.0 & 58.3 & 75.0 \\
\hline Extracapsular extension (\%) & 8.3 & 16.7 & 50.0 & 66.7 \\
\hline $\begin{array}{l}\text { Extracapsular extension }{ }^{\mathrm{a}, \mathrm{b}} \\
\text { (positive margins per mouse prostate) }\end{array}$ & $0.08 \pm 0.08$ & $0.33 \pm 0.22$ & $0.75 \pm 0.25$ & $1.83 \pm 0.46^{\mathrm{d}}$ \\
\hline Metastasis (\%) & 0 & 0 & 25.0 & 41.7 \\
\hline Metastasis $^{\mathrm{a}, \mathrm{c}}$ (sites per mouse) & 0 & 0 & $0.33 \pm 0.19$ & $2.75 \pm 1.09^{\mathrm{d}}$ \\
\hline
\end{tabular}

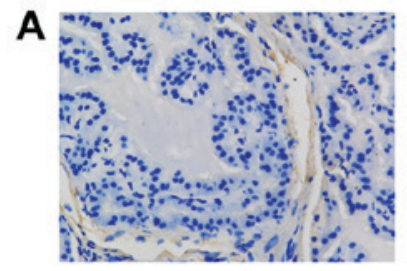

B
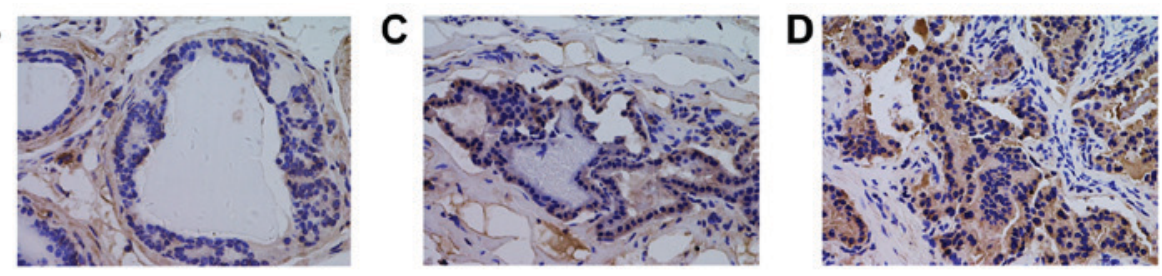

E

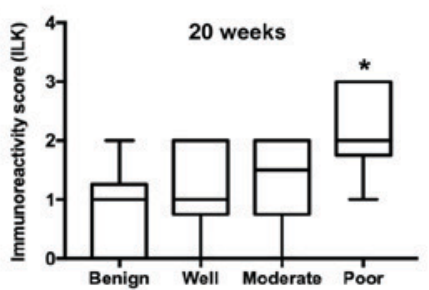

$\mathbf{F}$

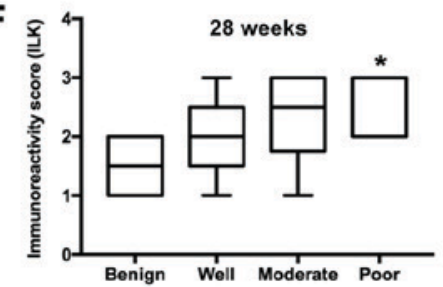

G

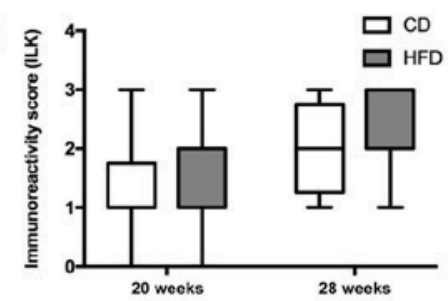

Figure 1. Representative IHC staining demonstrating ILK expression of different IRS category in TRAMP prostate and ILK levels compared among different prostate tissue and between different diet feeding. (A-D) Representatives images showing (A) negative (IRS category=0), (B) weak (IRS category=1), (C) moderate (IRS category=2) and (D) strong (IRS category=3) expressions of ILK in prostate specimens (magnification, $x 400$ ). (E and F) Boxplots graphs showing the IRS category of ILK expression across benign prostate tissue, well-differentiated, moderately-differentiated and poorly-differentiated prostate cancer in 20-week and 28-week TRAMP mice, respectively. The horizontal line indicates the median and the central box indicates the inter-quartile range, with whiskers indicate the lowest and highest results. ${ }^{*} \mathrm{P}<0.05$, significant differences are indicated as compared with benign subgroup. (G) Boxplots graphs showing the IRS category of ILK expression in prostate cancer between CD-fed and HFD-fed TRAMP mice of 20 and 28 weeks of age. The horizontal line and central box shows the median and inter-quartile range, with whiskers indicating the lowest and highest result. ILK, integrin-linked kinase; TRAMP, transgenic adenocarcinoma of mouse prostate; IHC, immunochemistry; CD, control diet; HFD, high-fat diet; IRS, immunoreactivity score.

CD-fed mice, there was a trend towards poorer PCa differentiation in HFD-fed mice, whereas no statistical significance was detected. Moreover, HFD-fed mice suffered higher rates of extracapsular extension (20-week, $16.7 \%$ vs. $8.3 \%$; 28 -week, $66.7 \%$ vs. $50.0 \%$ ), as well as higher rates of distant metastasis, eg, retroperitoneal lymph nodes or lung metastasis (28-week, $41.7 \%$ vs. $25.0 \%$ ). In quantitative analysis, the average positive margins of extracapsular extension and average sites of metastasis were both significantly higher in 28-week HFD-fed mice.

Protein expression of ILK, $\beta$-parvin and cofilin 1 in HFD-induced PCa progression. The representative IHC images of ILK, $\beta$-parvin and cofilin 1 in prostate specimens 
A

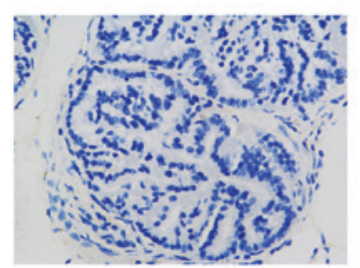

B

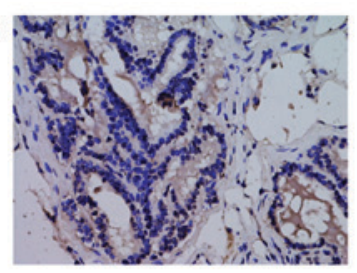

C

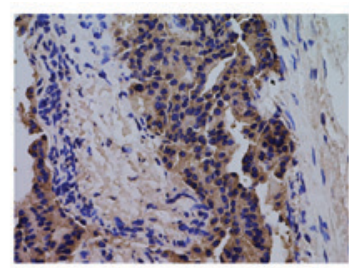

D

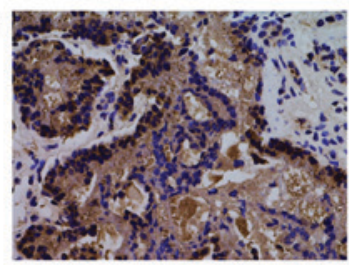

E

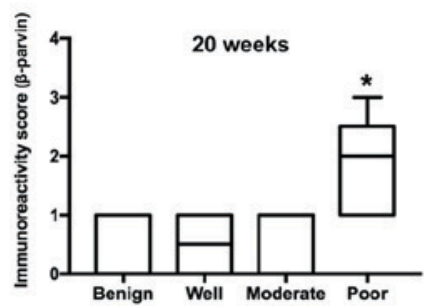

$\mathbf{F}$

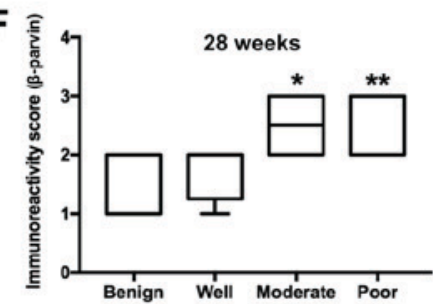

G

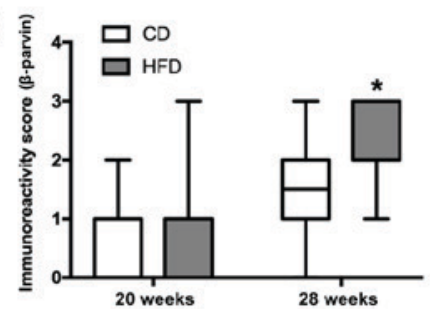

Figure 2. Representative IHC staining demonstrating $\beta$-parvin expression of different IRS category in TRAMP prostate and $\beta$-parvin levels compared among different prostate tissue and between different diet feeding. (A-D) Representatives images showing (A) negative (IRS category=0), (B) weak (IRS category=1), (C) moderate (IRS category=2) and (D) strong (IRS category=3) expressions of $\beta$-parvin in prostate specimens (magnification, $x 400$ ). (E and F) Boxplots graphs showing the IRS category of $\beta$-parvin expression across benign prostate tissue, well-differentiated, moderately-differentiated and poorly-differentiated prostate cancer in 20-week and 28-week TRAMP mice, respectively. The horizontal line indicates the median and the central box indicates the inter-quartile range, with whiskers indicate the lowest and highest results. ${ }^{*} \mathrm{P}<0.05,{ }^{* *} \mathrm{P}<0.01$, significant difference is indicated as compared with benign subgroup. (G) Boxplots graphs showing the IRS category of $\beta$-parvin expression in prostate cancer between CD-fed and HFD-fed TRAMP mice of 20 and 28 weeks of age. The horizontal line and central box shows the median and inter-quartile range, with whiskers indicating the lowest and highest result. ${ }^{*} \mathrm{P}<0.05$, significant difference is indicated as compared with control-diet fed mice of the same week subgroup. TRAMP, transgenic adenocarcinoma of mouse prostate; IHC, immunochemistry; CD, control diet; HFD, high-fat diet; IRS, immunoreactivity score.

\section{A}

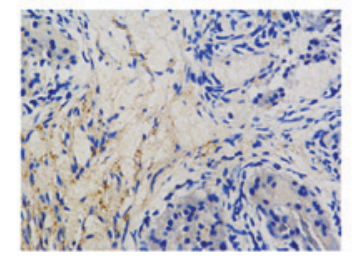

\section{B}

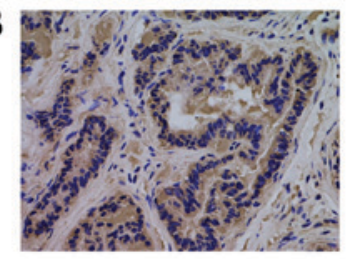

E

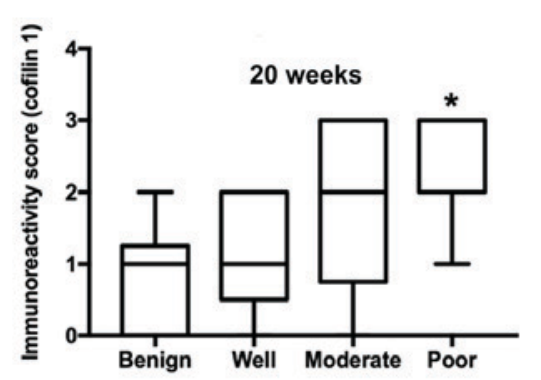

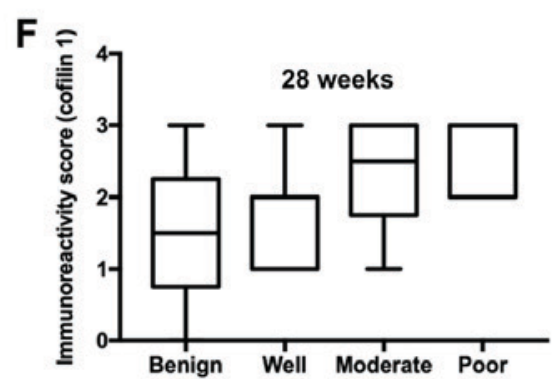
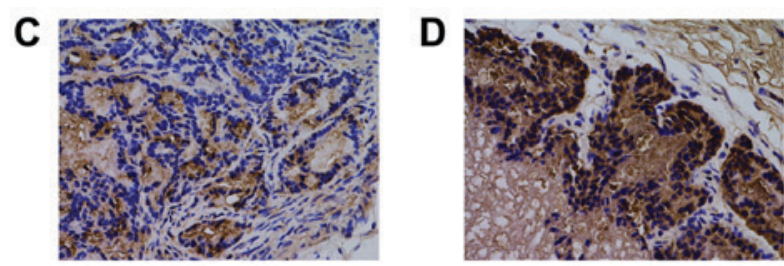

G

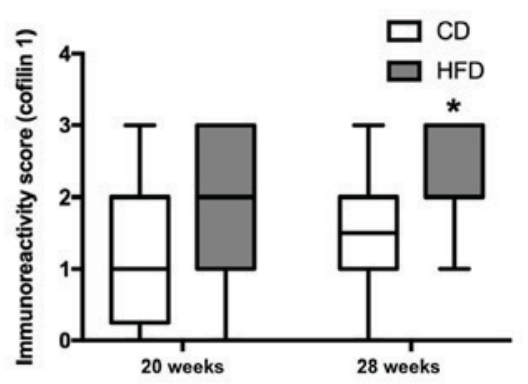

Figure 3. Representative IHC staining demonstrating cofilin 1 expression of different IRS category in TRAMP prostate and cofilin 1 levels compared among different prostate tissue and between different diet feeding. (A-D) Representatives images showing (A) negative (IRS category=0), (B) weak (IRS category=1), (C) moderate (IRS category=2) and (D) strong (IRS category=3) expression of cofilin 1 in prostate specimens (magnification, $\mathrm{x} 400$ ). (E and F) Boxplots graphs showing the IRS category of cofilin 1 expressions across benign prostate tissue, well-differentiated, moderately-differentiated and poorly-differentiated prostate cancer in 20-week and 28-week TRAMP mice, respectively. The horizontal line indicates the median and the central box indicates the inter-quartile range, with whiskers indicate the lowest and highest results. ${ }^{*} \mathrm{P}<0.05$, significant differences are indicated as compared with benign subgroup. (G) Boxplots graphs showing the IRS category of cofilin 1 expression in prostate cancer between CD-fed and HFD-fed TRAMP mice of 20 and 28 weeks of age. The horizontal line and central box shows the median and inter-quartile range, with whiskers indicating the lowest and highest result. ${ }^{*} \mathrm{P}<0.05$, significant difference is indicated as compared with control-diet fed mice of the same week subgroup. TRAMP, transgenic adenocarcinoma of mouse prostate; IHC, immunochemistry; CD, control diet; HFD, high-fat diet; IRS, immunoreactivity score.

(including benign prostate tissue and PCa), with IRS category ranging from 0-3 were presented as reference, respectively (Figs. 1-3). The staining for ILK, $\beta$-parvin and cofilin 1 was mainly located at cytoplasm.

Compared to benign prostate tissue, the ILK immunoreactivity was stronger in poorly-differentiated $\mathrm{PCa}$ in both 20-week and 28-week mice (Fig. 1E and F). Meanwhile, the expression of ILK presented with a slight increase in 28-week
HFD-fed mice (IRS category $=2.50 \pm 0.67$ vs. $2.00 \pm 0.74$, $\mathrm{P}=0.242$ ) (Fig. 1G) as compared with $\mathrm{CD}$-fed mice.

Immunoreactivity of $\beta$-parvin also increased steadily as $\mathrm{PCa}$ progressed, and was higher in poorly differentiated-PCa than that in benign prostate tissue (Fig. 2E and F). In 28 -week mice, the $\beta$-parvin expressions were also higher in HFD-fed group (IRS category $=2.25 \pm 0.62$ vs. $1.50 \pm 0.80$, $\mathrm{P}=0.038$ ) (Fig. 2G). 


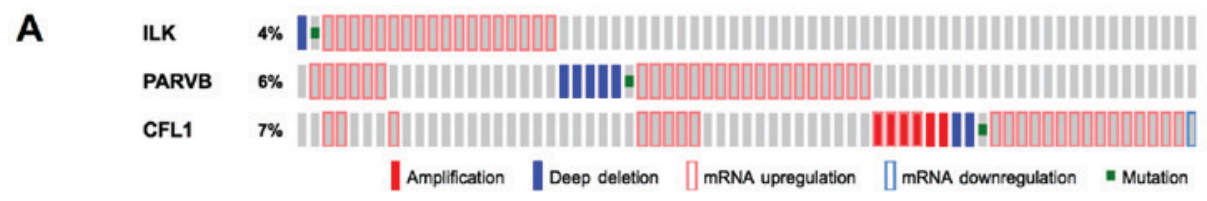

\begin{tabular}{|c|c|c|c|c|c|c|c|}
\hline Gene A & $\hat{\imath}$ & Gene B & $\hat{\imath}$ & P-value 8 - & $\underset{\text { ratio } \boldsymbol{\theta}}{\text { Log odds }}$ ? & Association (9) & \\
\hline ILK & & PARVB & & $<0.001$ & 2.077 & Tendency towards co-occurrence & Significant \\
\hline PARVB & & CFL1 & & 0.002 & 1.669 & Tendency towards co-occurrence & Significamt \\
\hline ILK & & CFL1 & & 0.134 & 0.989 & Tendency towards co-occurrence & \\
\hline
\end{tabular}
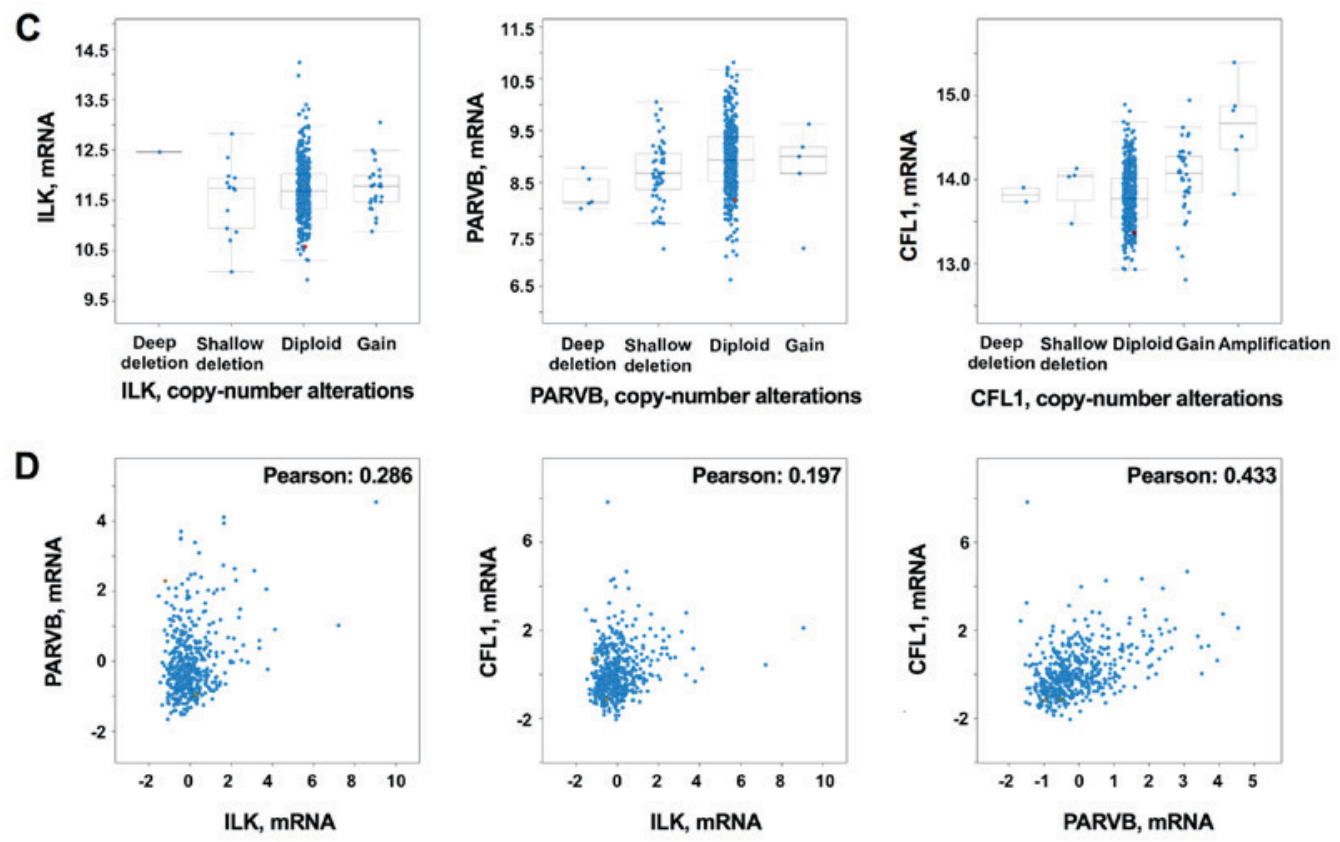

Figure 4. Protein and genetic expression profiles of $I L K, P A R V B, C F L 1$ in prostate adenocarcinoma from TCGA database. (A) Oncoprint of $I L K, P A R V B$ and $C F L 1$ genetic alterations, with the specific alteration categories presented in the lower panel. (B) The tendency towards co-occurrence among $I L K, P A R V B$ and $C F L 1$. (C) Plots showing the associations between DNA copy number alterations and related mRNA abundance in the gene ILK (left panel), PARVB (middle panel) and $C F L 1$ (right panel) in prostate adenocarcinoma. Blue dots indicate individual cases. (D) Plots demonstrating the mRNA expression correlations in $P A R V B$ vs. $I L K$ (left panel), $C F L 1$ vs. $I L K$ (middle panel) and $C F L 1$ vs. PARVB (right panel). Correlations are determined by Pearson coefficient with P-value. Blue dots indicate individual cases. TCGA, The Cancer Genome Atlas.

As for cofilin 1 expression, we identified a higher IRS category in poorly-differentiated $\mathrm{PCa}$ in 20 -week mice (Fig. 3E), while no significant difference was detected in 28-week mice (Fig. 3F). Besides, the cofilin 1 expressions were higher in 28-week HFD-fed group (IRS category $=2.50 \pm 0.67$ vs. $1.50 \pm 0.80, \mathrm{P}=0.018$ ) (Fig. $3 \mathrm{G}$ ).

ILK, PARVB and CFL1 jointly participated in PCa progression and correlated with worse disease-free survival within a public database. The genetic alterations in ILK, PARVB and CFLI were evaluated in TCGA database from 499 PCa samples. In total, $\sim 17 \%$ of PCa patients exhibited alterations (mainly in mRNA upregulation and amplification) in either ILK, PARVB or $C F L 1$ levels (Fig. 4A), with both mRNA and protein expression Z-score threshold \pm 2 . Both ILK-PARVB and PARVB-CFLI gene pairs showed tendencies towards co-occurrence (Fig. 4B). We further discovered that upregulation of gene ILK and $P A R V B$, as well as the upregulation and amplification of gene $C F L 1$ were all correlated with an increase in the corresponding mRNA (Fig. 4C). Besides, slight to moderate positive correlations were detected in three gene pairs (ILK-PARVB,
Pearson 0.286, $\mathrm{P}<0.001$; ILK-CFL1, Pearson 0.197, $\mathrm{P}<0.001$; PARVB-CFL1, Pearson 0.433, P<0.00) (Fig. 4D).

In the analysis of DFS, a total of 499 PCa patients from TCGA provisional database were enrolled. Among them, 92 (18.4\%) patients suffered disease recurrence and progression. DFS was compared between subgroups expressing higher vs. lower levels of mRNA (75th and 25th percentile as cutoff) (Fig. 5). In the whole cohort, a tendency towards worse DFS was observed in the patients with higher CFL1 mRNA expression (Log-Rank $\mathrm{P}=0.083$ ) (Fig. 5A). In subgroup analysis, higher mRNA expression in CFL1 was correlated with worse DFS (Log-Rank $\mathrm{P}=0.048$ ) (Fig. 5B) in patients with stage III and IV PCa. Meanwhile, in patients with Gleason score $\geq 7$, trends towards worse DFS were also detected in patients with higher mRNA expression of PARVB (Log-Rank $\mathrm{P}=0.095$ ) (Fig. 5C) and CFL1 (Log-Rank $\mathrm{P}=0.064$ ) (Fig. 5C).

\section{Discussion}

Increasing evidence indicated a positive association between obesity and PCa incidence and aggressiveness (15). Generally, 

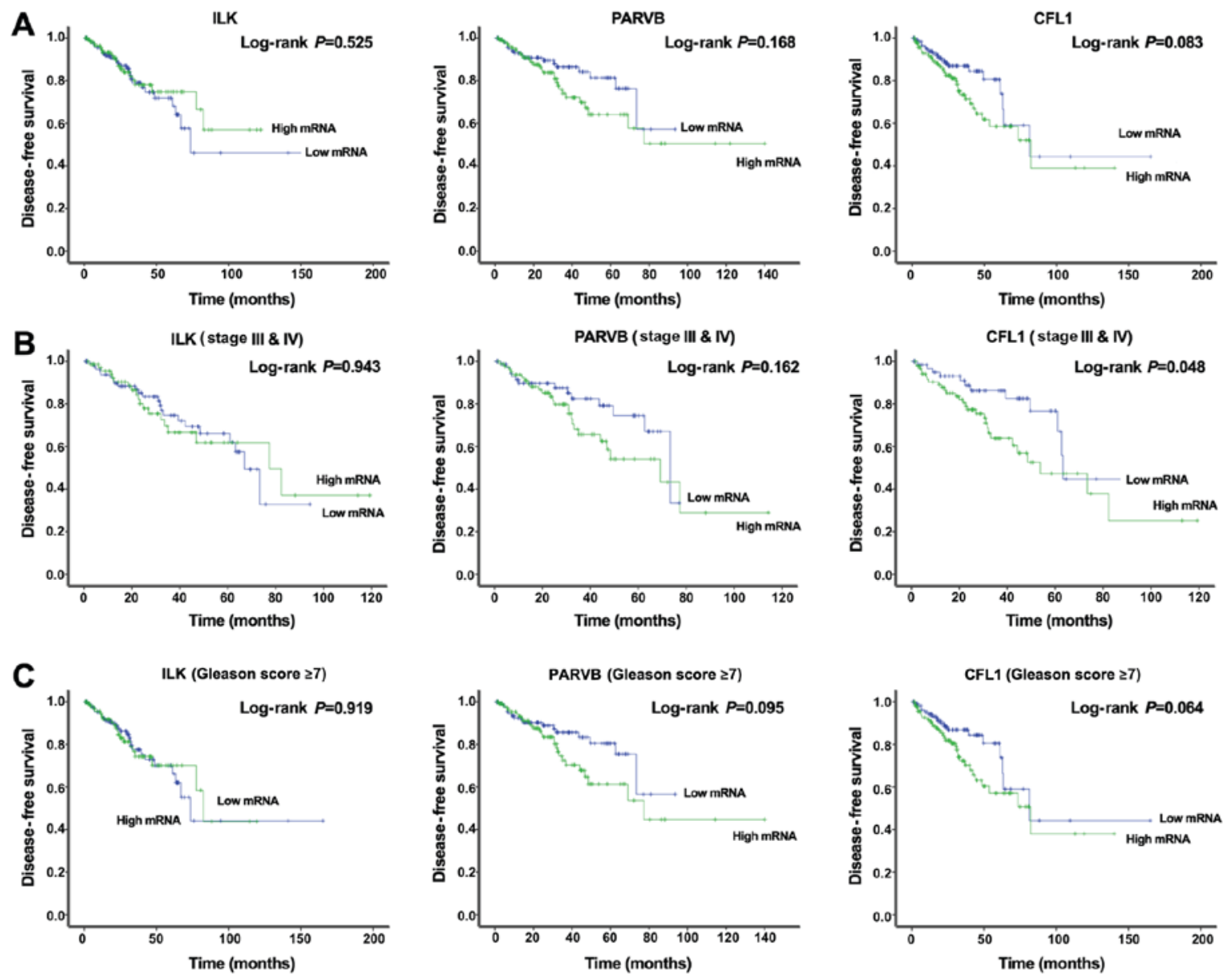

Figure 5. Effects of mRNA expression of $I L K, P A R V B$ and $C F L 1$ on the disease-free survival in TCGA prostate adenocarcinoma cohort. (A) Kaplan-Meier plots with log-rank test demonstrating the disease-free survival in patients expressing high vs. low mRNA levels of $I L K$ (left panel), $P A R V B$ (middle panel) and $C F L 1$ (right panel). (B) Disease-free survival curves for stage III and stage IV cancer patients with high vs. low mRNA expression in ILK (left panel), $P A R V B$ (middle panel) and CFL1 (right panel). (C) Disease-free survival curves for Gleason score $\geq 7$ cancer patients with high vs. low mRNA expression in $I L K$ (left panel), PARVB (middle panel) and CFLl (right panel). TCGA, The Cancer Genome Atlas.

the positive association was due to three major mechanisms, including decreased testosterone, adipokine alterations and insulin resistance $(2,16)$. Recently, several studies reported the involvement of ILK, $\beta$-parvin and cofilin in patients with obesity or metabolic syndromes (7-9). Meanwhile, a pioneering research conducted by Shibue et al firstly identified that the activation of ILK/ $\beta$-parvin/cofilin pathway was critical in the formation of FLPs and the progression of carcinomas (6). Therefore, the present study aimed to validate the expression of ILK, $\beta$-parvin and cofilin in PCa in TRAMP model, and to further explore the role of obesity induced protein expression alterations in the progression of PCa.

Dietary high fat was proved to induce obesity by increasing fat deposit in body, and in turn, the excessive fat would further affect cancer progression (17). Several studies were conducted in murine xenograft $(18,19)$ and genetically engineered animal models (20), and discovered that HFD-fed would cause obesity and promote $\mathrm{PCa}$ development and progression. Among them, the application of genetically engineered mouse (GEM) caught great attention from researchers. Till now, four kinds of GEM model (TRAMP, LADY, Hi-Myc, Pten-null) were widely used (21). Among them, the TRAMP model was the first and also one of the most widely used model.
The TRAMP model could successfully recapitulate all the parameters of PCa progression in human, including formation and progression from prostatic hyperplasia, intraepithelial neoplasia, adenocarcinoma to metastatic PCa (22). Besides, the great frequency and breadth of metastases was another merit of TRAMP in the research of cancer aggressiveness. The TRAMP model was specifically appreciated in the research of tumor microenvironment-cancer progression relationship, as neoplastic prostates were marked by stromal remodeling (reactive stroma) (22). Consistent with previous studies, the present study demonstrated that HFD feeding increased body weight and adipose tissue deposit in TRAMP mice, and HFD-fed mice possessed PCa with poorer differentiation, higher rates of extracapsular extension and metastasis, especially in 28 -week group.

In the present study, ILK (encoded by gene $I L K$ ) was proved to be overexpressed in PCa, especially in poorly-differentiated $\mathrm{PCa}$, which was consistent with previous outcomes in breast cancer, melanoma, colon cancer and PCa (23). Several in vivo studies showed the role of ILK in different aspects of cancer progression, including cell growth, EMT, migration and invasion (24). We also detected a slight increase of ILK expression in HFD-fed mice, though without statistical significance, and the outcomes warranted further investigation. 
$\beta$-parvin (encoded by PARVB) linked with ILK and constituted IPP complex, which was known to facilitate FLPs formation, promote cell motility and extracellular matrix adhesion $(6,25)$. Of note, the present study for the first time described the expression of $\beta$-parvin in $\mathrm{PCa}$, and identified its overexpression in poorly-differentiated PCa. Moreover, we identified that HFD feeding could increase the expression of $\beta$-parvin in PCa. From TCGA database, we further discovered a trend towards worse DFS in PCa patients (Gleason score $\geq 7$ ) with higher mRNA expression of $P A R V B$. Previous studies reported conflicting outcomes, as $P A R V B$ was downregulated in breast cancer (26) and urothelial cell carcinoma (27), while overexpressed and correlated with tumor progression in colorectal cancer (12) and tongue squamous cell carcinoma (28), which implied an organ-specific expression or function of $\beta$-parvin.

Cofilin 1 (encoded by $C F L 1$ ), an actin binding protein that played an key role in actin filament dynamics, functioned in cancer cell migration, invasion and mitosis (29). The present study found that cofilin 1 was overexpressed in moderately and poorly-differentiated PCa of TRAMP mice, which was in consistency with previous study (30). From TCGA database, higher mRNA expression of CFL1 was considered a risk factor for worse DFS in patients with Stage III or IV PCa. In HFD-fed group, we detected an increase in cofilin 1 expression.

In TCGA prostate adenocarcinoma database, ILK, PARVB and $C F L 1$ all presented mainly with mRNA upregulation. A tendency towards co-occurrence was also identified in gene pairs of ILK-PARVB and PARVB-CFL1. These observations implied close relationships among these genes and the possible mechanisms contributed to carcinogenesis in their neighborhood network.

Several limitations existed in the present study. First, the key objects (ILK, $\beta$-parvin and cofilin 1) was only examined and evaluated by IHC, which required further western blotting and reverse transcription polymerase chain reaction study to confirm. Second, the alterations in the level of these proteins might result from both the diet and the progression of $\mathrm{PCa}$. The discrimination of these two factors was difficult. Third, the TCGA database did not contain obesity, BMI, or diet habit as a clinical parameter, so we failed to apply these factors in the stratified analysis. Besides, the K-M survival analysis from TCGA database could not take elemental parameters (age, race) into account during analysis, therefore the associations between the subject proteins and patient survival were warranted by more cohort studies.

In conclusion, the present study demonstrated that HFD feeding contributed to higher expressions of $\beta$-parvin and cofilin 1 in $\mathrm{PCa}$ tissue and promoted cancer progression in TRAMP mice. Besieds, higher expressions of ILK, $\beta$-parvin and cofilin 1 were associated with poorer cancer differentiation. In TCGA database, higher mRNA levels of CFL1 were identified to be correlated with worse disease-free survival in certain subgroups. Further studies were warranted in discussing the potential roles of ILK, $\beta$-parvin and cofilin 1 in HFD feeding induced progression of PCa.

\section{Acknowledgements}

Not applicable.

\section{Funding}

The present study was supported by the Shanghai Sailing Program (grant no. 17YF1401700) and the National Natural Science Foundation of China (grant no. 81272835). The funders had no role in study design, data collection and analysis, decision to publish or preparation of the manuscript.

\section{Availability of data and materials}

The datasets used and/or analyzed during the current study are available from the corresponding author on reasonable request.

\section{Authors' contributions}

MBH, JMH, TY, WHZ, YH and XBW performed the animal studies and histological staining. LRJ performed the pathological analysis. MBH performed the general statistical analysis and drafted the manuscript. HWJ and QD conceived the study and participated in its design and coordination. All authors read and approved the final manuscript.

\section{Ethics approval and consent to participate}

The present study was performed in strict accordance with the National Institutes of Health guide for the care and use of Laboratory animals (NIH publication no. 8023, revised 1978). The protocol was approved by the Institutional Animal Care and Use Committee from Department of Laboratory Animal Science, Fudan University (approval no. 20160816A197).

\section{Patient consent for publication}

Not applicable.

\section{Competing interests}

The authors declare that they have no competing interests.

\section{References}

1. Torre LA, Bray F, Siegel RL, Ferlay J, Lortet-Tieulent J and Jemal A: Global cancer statistics, 2012. CA Cancer J Clin 65: 87-108, 2015.

2. Parikesit D, Mochtar CA, Umbas R and Hamid AR: The impact of obesity towards prostate diseases. Prostate Int 4: 1-6, 2016.

3. Hu MB, Liu SH, Jiang HW, Bai PD and Ding Q: Obesity affects the biopsy-mediated detection of prostate cancer, particularly high-grade prostate cancer: A dose-response meta-analysis of 29,464 patients. PLoS One 9: e106677, 2014.

4. Hu MB, Xu H, Bai PD, Jiang HW and Ding Q: Obesity has multifaceted impact on biochemical recurrence of prostate cancer: A dose-response meta-analysis of 36,927 patients. Med Oncol 31: 829, 2014

5. Calle EE, Rodriguez C, Walker-Thurmond $\mathrm{K}$ and Thun MJ: Overweight, obesity, and mortality from cancer in a prospectively studied cohort of U.S. adults. N Engl J Med 348: 1625-1638, 2003.

6. Shibue T, Brooks MW and Weinberg RA: An integrin-linked machinery of cytoskeletal regulation that enables experimental tumor initiation and metastatic colonization. Cancer Cell 24: 481-498, 2013.

7. Liu Z, Xiao Y, Yuan Y, Zhang X, Qin C, Xie J, Hao Y, Xu T and Wang X: Effects of oleic acid on cell proliferation through an integrin-linked kinase signaling pathway in 786-O renal cell carcinoma cells. Oncol Lett 5: 1395-1399, 2013. 
8. Kang L, Mokshagundam S, Reuter B, Lark DS, Sneddon CC, Hennayake C, Williams AS, Bracy DP, James FD, Pozzi A, et al: Integrin-linked kinase in muscle is necessary for the development of insulin resistance in diet-induced obese mice. Diabetes 65: 1590-1600, 2016.

9. Tabur S, Oztuzcu S, Oguz E, Demiryürek S, Dagli H, Alasehirli B, Ozkaya $\mathrm{M}$ and Demiryürek AT: Evidence for elevated (LIMK2 and CFL1) and suppressed (ICAM1,EZR, MAP2K2 and NOS3) gene expressions in metabolic syndrome. Endocrine 53: 465-470, 2016.

10. Nguewa PA and Calvo A: Use of transgenic mice as models for prostate cancer chemoprevention. Curr Mol Med 10: 705-718, 2010.

11. Xu H, Hu MB, Bai PD, Zhu WH, Liu SH, Hou JY, Xiong ZQ, Ding Q and Jiang HW: Proinflammatory cytokines in prostate cancer development and progression promoted by high-fat diet. Biomed Res Int 2015: 249741-7, 2015.

12. Bravou V, Antonacopoulou A, Papanikolaou S, Nikou S, Lilis I, Giannopoulou E and Kalofonos HP: Focal adhesion proteins $\alpha$ - and $\beta$-Parvin are overexpressed in human colorectal cancer and correlate with tumor progression. Cancer Invest 33: 387-397, 2015.

13. Gao J, Aksoy BA, Dogrusoz U, Dresdner G, Gross B, Sumer SO, Sun Y, Jacobsen A, Sinha R, Larsson E, et al: Integrative analysis of complex cancer genomics and clinical profiles using the cBioPortal. Sci Signal 6: pl1, 2013.

14. Cerami E, Gao J, Dogrusoz U, Gross BE, Sumer SO, Aksoy BA, Jacobsen A, Byrne CJ, Heuer ML, Larsson E, et al: The cBio cancer genomics portal: An open platform for exploring multidimensional cancer genomics data. Cancer Discov 2: 401-404, 2012.

15. Center MM, Jemal A, Lortet-Tieulent J, Ward E, Ferlay J, Brawley $\mathrm{O}$ and Bray F: International variation in prostate cancer incidence and mortality rates. Eur Urol 61: 1079-1092, 2012

16. Allott EH, Masko EM and Freedland SJ: Obesity and prostate cancer: Weighing the evidence. Eur Urol 63: 800-809, 2013.

17. Toren P, Mora BC and Venkateswaran V: Diet, obesity and cancer progression: Are adipocytes the link? LPI 6: 37-45, 2013.

18. Mavropoulos JC, Buschemeyer WC III, Tewari AK, Rokhfeld D, Pollak M, Zhao Y, Febbo PG, Cohen P, Hwang D, Devi G, et al: The effects of varying dietary carbohydrate and fat content on survival in a murine LNCaP prostate cancer xenograft model. Cancer Prev Res (Phila) 2: 557-565, 2009.

19. Cho HJ, Kwon GT, Park H, Song H, Lee KW, Kim JI and Park JHY: A high-fat diet containing lard accelerates prostate cancer progression and reduces survival rate in mice: Possible contribution of adipose tissue-derived cytokines. Nutrients 7 $2539-2561,2015$
20. Kobayashi N, Barnard RJ, Said J, Hong-Gonzalez J, Corman DM, Ku M, Doan NB, Gui D, Elashoff D, Cohen P and Aronson WJ: Effect of low-fat diet on development of prostate cancer and Akt phosphorylation in the Hi-Myc transgenic mouse model. Cancer Res 68: 3066-3073, 2008.

21. Bianchi-Frias D, Hernandez SA, Coleman R, Wu H and Nelson PS: The landscape of somatic chromosomal copy number aberrations in GEM models of prostate carcinoma. Mol Cancer Res 13: 339-347, 2015.

22. Gelman IH: How the TRAMP model revolutionized the study of prostate cancer progression. Cancer Res 76: 6137-6139, 2016.

23. Graff JR, Deddens JA, Konicek BW, Colligan BM, Hurst BM, Carter HW and Carter JH: Integrin-linked kinase expression increases with prostate tumor grade. Clin Cancer Res 7: 1987-1991, 2001

24. Cortez V, Nair BC, Chakravarty D and Vadlamudi RK: Integrin-linked kinase 1: Role in hormonal cancer progression. Front Biosci (Schol Ed) 3: 788-796, 2011.

25. Sepulveda JL and Wu C: The parvins. Cell Mol Life Sci 63: 25-35, 2006.

26. Mongroo PS, Johnstone CN, Naruszewicz I, Leung-Hagesteijn C, Sung RK, Carnio L, Rustgi AK and Hannigan GE: Beta-parvin inhibits integrin-linked kinase signaling and is downregulated in breast cancer. Oncogene 23: 8959-8970, 2004.

27. Wu CF, Ng KF, Chen CS, Chang PL, Chuang CK, Weng WH, Liao SK and Pang ST: Expression of parvin-beta is a prognostic factor for patients with urothelial cell carcinoma of the upper urinary tract. Br J Cancer 103: 852-860, 2010.

28. Eslami A, Miyaguchi K, Mogushi K, Watanabe H, Okada N, Shibuya H, Mizushima H, Miura M and Tanaka H: PARVB overexpression increases cell migration capability and defines high risk for endophytic growth and metastasis in tongue squamous cell carcinoma. Br J Cancer 112: 338-344, 2015.

29. Mizuno K: Signaling mechanisms and functional roles of cofilin phosphorylation and dephosphorylation. Cell Signal 25: 457-469, 2013.

30. Lu LI, Fu NI, Luo XU, Li XY and Li XP: Overexpression of cofilin 1 in prostate cancer and the corresponding clinical implications. Oncol Lett 9: 2757-2761, 2015.

This work is licensed under a Creative Commons Attribution-NonCommercial-NoDerivatives 4.0 International (CC BY-NC-ND 4.0) License. 